\title{
Crianças do Pré-Escolar a Resolver Problemas de Estrutura Aditiva: Que estratégias?
}

\section{Kindergarten Children Solving Additive Problems: Which Strategies?}

\author{
Florbela Soutinho* \\ ORCID iD 0000-0002-6136-2107 \\ Ema Mamede** \\ ORCID iD 0000-0002-1623-8406
}

\begin{abstract}
Resumo
Os estudos desenvolvidos em diferentes contextos ressaltam a capacidade que as crianças têm de resolver corretamente problemas de adição e subtração, antes ainda destas operações lhes serem formalmente ensinadas. $\mathrm{O}$ estudo aqui descrito procura perceber como as crianças dos 4 aos 6 anos $(\mathrm{N}=90)$ entendem os problemas de estrutura aditiva. Para tal, tenta responder às seguintes questões: 1) Que desempenhos apresentam as crianças quando resolvem problemas de estrutura aditiva? 2) Que estratégias usam para resolver os problemas de estrutura aditiva? Adotou-se uma metodologia quantitativa que analisa os desempenhos e as estratégias das crianças quando resolvem 28 problemas de estrutura aditiva, apresentados a partir de entrevistas estruturadas individuais. Os resultados sugerem que as crianças resolvem com facilidade os problemas propostos e utilizam estratégias adequadas para responderem corretamente, chegando mesmo a recorrer a estratégias abstratas como a contagem e os fatos numéricos.
\end{abstract}

Palavras-chave: Resolução de Problemas. Estrutura Aditiva. Estratégias de Resolução.

\begin{abstract}
Several studies report young children's ability to solve addition and subtraction problems before receiving any formal instruction. This study focuses on how 4-6-year-old children $(\mathrm{N}=90)$ understand additive structure problems. It addresses two questions: 1) How do children perform when solving additive structure problems? 2) What strategies do children use when solving additive structure problems? Quantitative methods were used to analyse children's performance and strategies when solving 28 additive structure problems presented to them using individual interviews. Results suggest that children easily solved the given problems using adequate strategies, some could even count and rely on numerical facts.
\end{abstract}

Keywords: Problem Solving. Additive Structure. Solving Strategies.

\section{Conhecimento informal}

\footnotetext{
* Doutora, Universidade do Minho (UMinho). Colaboradora, CIEC, Universidade do Minho (UMinho), Braga, Portugal. Endereço para correspondência: Rua do Olival, lote 5, Orgens, Viseu, Portugal, CEP: 3510-674. Email: soutinhoflorbela@gmail.com.

** Doutora, Universidade do Minho (UMinho)/OBU. Professora Auxiliar, CIEC, Universidade do Minho (UMinho), Braga, Portugal. Endereço para correspondência: Instituto de Educação, Universidade do Minho, Campus de Gualtar, Braga, Portugal, CEP: 4710-057. E-mail: emamede@ie.uminho.pt.
} 
Durante a idade pré-escolar, as crianças desenvolvem uma grande quantidade de conhecimento como resultado da sua experiência diária, entendido como conhecimento informal. Não desconsiderando o conhecimento informal como o que é desenvolvido no dia a dia, fora da escola, ou mesmo na escola sem ser "ensinado" (BECKER \& SELTER, 1994), adota-se aqui a definição de Baroody e Ginsburg (1986), que se referem ao conhecimento informal como aquele que a criança desenvolve diariamente, antes da escolaridade formal, e que mais tarde transporta para o processo de aprendizagem. Alguns autores sugerem que a Educação Matemática deve ser construída a partir do conhecimento informal das crianças (ver BAROODY \& GINSBURG, 1986; RESNICK, 1989). Este conhecimento informal é de tal forma rico que Nunes, Bryant e Watson (2009) sugerem mesmo que alguns dos modelos intuitivos que as crianças usam traduzem a compreensão de princípios lógicos que lhes permitem a apropriação de noções matemáticas.

Ginsburg e Seo (1999) entendem que a Matemática informal das crianças contém, num nível implícito, muitas das ideias matemáticas que os professores precisam promover num nível formal e explícito. Resnick (1989) afirma que uma forma de perceber o desenvolvimento do conceito do número na criança é analisar o recurso à contagem nas estratégias informais e inventadas quando resolvem problemas. Quando a criança deixa de "contar todos" para "contar a partir de" é um sinal de que as crianças relacionam o seu conhecimento das relações parte-todo com a sequência de contagem, demonstrando uma compreensão inicial da composição aditiva do número (NUNES; BRYANT; WATSON, 2009). Assim, o modo como as crianças usam a contagem na resolução de problemas de estrutura aditiva reflete as alterações que se vão produzindo no seu raciocínio.

\section{Sobre o raciocínio aditivo}

De acordo com Vergnaud (1982), o campo conceitual das estruturas aditivas é simultaneamente o conjunto das situações cujo tratamento implica uma ou mais adições e subtrações e o conjunto dos conceitos e procedimentos que possibilitam a análise dessas situações, enquanto tarefas matemáticas. $\mathrm{O}$ raciocínio aditivo diz respeito à análise lógica de problemas que envolvem a adição e subtração, cujos conceitos-chave implícitos se relacionam com a composição aditiva e com a relação inversa entre adição e subtração (NUNES \& BRYANT, 1996; NUNES; BRYANT; WATSON, 2009).

O tipo de conhecimento que as crianças desenvolvem inicialmente parece estar relacionado com três tipos de ação: juntar mais elementos a um conjunto, retirar elementos de 
um conjunto, colocar em correspondência termo-a-termo os elementos de dois conjuntos (NUNES \& BRYANT, 1996).

Segundo Piaget e Szeminska (1971), as crianças desenvolvem os esquemas de juntar e separar como independentes um do outro, sem compreender a relação que existe entre os dois, e conseguem uma compreensão mais avançada quando passam do conhecimento baseado em esquemas de ação para um conceito operatório de adição e subtração, coordenando os dois esquemas de forma a reconhecerem a relação inversa entre a adição e a subtração. Para Nunes, Bryant e Watson (2009), esta coordenação dos esquemas de ação é essencial à construção do conceito de adição e subtração, necessários à resolução de problemas complexos. Perceber que a adição e a subtração da mesma quantidade deixa a quantidade inicial inalterada é essencial no raciocínio aditivo das crianças.

Considerando que todas as situações nas quais são usadas a adição ou subtração se inserem no campo conceitual das estruturas aditivas, Vergnaud (2011) alerta para situações delicadas, em que o sentido do enunciado não conduz de imediato a operação necessária à resolução do problema, indiciando, por vezes, uma ideia contrária da que está primariamente ligada à operação, como, por exemplo, realizar uma adição quando no enunciado é referido uma perda.

Desta forma, Vergnaud (2011) distingue situações aditivas mais claras e mais fáceis do que outras, discriminando situações prototípicas e não prototípicas. As situações prototípicas caracterizam-se por requererem a operação que está mencionada no problema, são situações que expressam claramente o sentido da operação; as não prototípicas caracterizam-se por requererem a operação inversa a que é mencionada no problema. $\mathrm{Na}$ adição existem duas situações prototípicas através das quais as crianças dão um primeiro sentido a esta operação: i) a reunião de duas partes num todo; ii) a transformação de uma quantidade inicial que resulta numa quantidade final. Estas duas situações são as mais simples, contudo, elas dão origem a situações mais complexas e que requerem, na sua resolução, uma operação contrária à que é descrita no problema. Situações em que se conhece o todo e uma das partes e se procura identificar a outra parte, ou situações de transformação em que se desconhece o estado inicial, obriga à solução pela operação contrária à que é descrita no enunciado, conduzindo a uma divergência de pensamento e ao conflito com as situações prototípicas da adição e subtração.

Para que ambas as situações façam sentido para as crianças como situações aditivas e adquiram significado enquanto tal, é necessário trabalhar com as crianças, tanto situações prototípicas, como as não prototípicas. Assim, entendendo as relações que são descritas nos problemas, as crianças analisam corretamente as situações e resolvem-nas adquadamente. 


\section{As estratégias de resolução dos problemas de estrutura aditiva}

As crianças usam estratégias diversificadas para resolver os problemas de estrutura aditiva, interpretando a situação que é descrita nos problemas. A literatura tem vindo a apresentar estudos que documentam vários tipos de estratégias observadas em crianças de diferentes idades na resolução de problemas de estrutura aditiva.

Carpenter e Moser (1984) referem que crianças americanas dos 6 aos 8 anos resolvem problemas de adição e subtração sem nunca terem recebido instrução formal sobre as operações. Observaram que algumas estratégias modelam naturalmente a situação que é descrita no problema. Os problemas de separar são maioritariamente resolvidos pela estratégia de Separar, assim como os problemas que têm subjacente a ação de juntar são resolvidos pela estratégia Juntar. Os problemas de comparação são resolvidos pela estratégia de Correspondência.

Também De Corte e Verschaffel (1987) observaram, num estudo que desenvolveram com 30 crianças de 6 anos, que as estratégias usadas são fortemente influenciadas quer pela estrutura semântica dos problemas, quer pela ordem em que são apresentadas as quantidades no enunciado dos problemas.

De igual modo, Fuson (1992) constata que crianças entre os 7 e 8 anos vão evoluindo no uso de estratégias. A autora distingue quatro níveis de estratégias: Sequência Inquebrável (contagem como uma cadeia de palavras sem quebras); Cadeia Quebrada (construção de relações entre as partes e o todo, separando as quantidades em partes); Cadeia Numérica (uso de contagem, como uma cadeia que se pode interromper); Cadeia Bidirecional (partindo de um dos números dados, prosseguem a contagem, sendo que posteriormente a sequência numérica torna-se ela própria o objeto presente nos termos da adição e da subtração).

Kamii e Dominick (1997) realçam que as crianças inventam procedimentos para resolver problemas de adição e subtração e sugerem que o uso de algoritmos as desencoraja a desenvolver o sentido de número, obrigando-as a desistirem da sua própria maneira de pensar. De estudos com crianças entre os 8 e 10 anos, os autores depreendem que quando estas pensam por si próprias estabelecem as relações mentais necessárias à construção do sentido de número e avançam na construção de níveis de pensamento mais elaborados.

Carpenter, Fennema, Levi e Empson (1999) consideram que inicialmente as crianças recorrem a estratégias de manipulação direta, com a representação física de cada quantidade envolvida no problema, passando depois a estratégias de contagem, em que a resposta se foca na própria sequência de contagem e, por último, fazem uso de estratégias mentais, com 
recurso a fatos conhecidos previamente memorizados, não manipulando objetos nem usando a contagem para resolver os problemas propostos. Carpenter e seus colaboradores observam que as crianças em idade pré-escolar modelam as ações e relações descritas nos problemas e usam estratégias distintas consoante cada um deles. Estes autores defendem mesmo que conceber a resolução dos problemas pela modelação das ações que lhe estão inerentes permite uma melhor estruturação do pensamento sobre a resolução dos problemas, nos primeiros anos de escolaridade. Além disso, ajuda a entender em que aspecto reside a dificuldade das crianças na resolução de determinados problemas que parecem simples aos olhos dos adultos.

Dessa forma, parece ser claro que as crianças a partir dos 6-7 anos conseguem resolver, com sucesso, alguns problemas de estrutura aditiva, com uma grande variedade de estratégias, algumas até com grande nível de abstração. Contudo, ainda não se conhece, com grande detalhe, como é que as crianças com idades anteriores resolvem os problemas de estrutura aditiva.

É objetivo deste estudo perceber como raciocinam as crianças de 4, 5 e 6 anos quando resolvem alguns problemas de estrutura aditiva. Para tal, tenta-se responder às questões: 1) Que desempenhos apresentam as crianças quando resolvem problemas de estrutura aditiva? 2) Que estratégias usam para os resolver?

\section{Metodologia}

Participaram no estudo 90 crianças dos 4 aos 6 anos ( 4 anos, $\mathrm{n}=30 ; 5$ anos, $\mathrm{n}=30 ; 6$ anos, $\mathrm{n}=30$ ), que frequentam a educação pré-escolar da rede pública, em Portugal, em escolas dos distritos de Viseu e Aveiro. Aos participantes foi aplicada uma entrevista estruturada, composta por 28 problemas de raciocínio aditivo, distribuídos equitativamente por duas sessões. Previamente realizaram-se testes-piloto para validar a entrevista no que respeita à sua extensão e aos tipos de problemas a apresentar às crianças.

Cada problema apresentava apenas uma questão e requeria a justificação de resposta. Os problemas propostos foram selecionados, adotando a classificação de Vergnaud (1982), tendo-se distinguido e apresentado 4 problemas de Composição de Duas Medidas, 12 de Transformação Ligando Duas Medidas e 12 de Relação Estática Ligando Duas Medidas. Em cada tipo de problemas atendeu-se ao elemento desconhecido e à direção da transformação ou da relação presente nos problemas. A Tabela 1 apresenta um exemplo de cada tipo de problemas apresentados às crianças. 
Tabela 1 - Exemplo de problemas de estrutura aditiva apresentados às crianças

\begin{tabular}{|c|c|c|c|}
\hline \multicolumn{4}{|c|}{$\begin{array}{l}\text { PROBLEMAS DE ESTRUTURA ADITIVA } \\
\end{array}$} \\
\hline $\begin{array}{l}\text { Tipo de } \\
\text { Problema }\end{array}$ & \multicolumn{2}{|c|}{$\begin{array}{l}\text { Elemento Desconhecido/ } \\
\text { Direção } \\
\end{array}$} & Exemplo \\
\hline \multirow{2}{*}{$\begin{array}{l}\text { Composição de } \\
\text { Duas Medidas }\end{array}$} & \multicolumn{2}{|l|}{ Todo } & $\begin{array}{l}\text { A cadelinha da Inês teve cachorros: } 5 \text { brancos e } 3 \text { castanhos. } \\
\text { Quantos cachorros teve, ao todo, a cadelinha da Inês? }\end{array}$ \\
\hline & \multicolumn{2}{|l|}{ Parte } & $\begin{array}{l}\text { O Paulo tem } 7 \text { brinquedos, } 5 \text { estão fora da caixa. Quantos estão } \\
\text { dentro da caixa? }\end{array}$ \\
\hline \multirow{6}{*}{$\begin{array}{l}\text { Transformação } \\
\text { Ligando Duas } \\
\text { Medidas }\end{array}$} & \multirow[t]{2}{*}{ Resultado } & Adição & $\begin{array}{l}\text { A mãe da Francisca deu-lhe } 4 \text { coelhinhos de chocolate. Mais } \\
\text { tarde deu-lhe mais 3. Quantos coelhinhos tem agora a Francisca? }\end{array}$ \\
\hline & & Subtração & O Rui tinha 7 rebuçados, deu 5 à sua irmã. Quantos tem agora? \\
\hline & \multirow{2}{*}{ Transformação } & Adição & $\begin{array}{l}\text { O bibe da Maria tinha } 4 \text { botões. A mãe coseu mais alguns. } \\
\text { Agora o bibe tem } 6 \text {. Quantos botões coseu a mãe? }\end{array}$ \\
\hline & & Subtração & $\begin{array}{l}\text { O Paulo tinha } 5 \text { rebuçados, comeu alguns e ficou com } 3 . \\
\text { Quantos rebuçados comeu? }\end{array}$ \\
\hline & \multirow{2}{*}{ Início } & Adição & $\begin{array}{l}\text { A Joana tinha algumas bonecas, a tia deu-lhe mais } 3 \text { e agora ela } \\
\text { tem } 8 \text {. Quantas bonecas tinha a Joana no início? }\end{array}$ \\
\hline & & Subtração & $\begin{array}{l}\text { A Ana tinha alguns rebuçados, deu } 3 \text { à sua mãe e ficou com } 2 . \\
\text { Quantos rebuçados tinha a Ana no início? }\end{array}$ \\
\hline \multirow{6}{*}{$\begin{array}{l}\text { Relação Estática } \\
\text { Ligando Duas } \\
\text { Medidas }\end{array}$} & \multirow{2}{*}{ Diferença } & Adição & $\begin{array}{l}\text { Numa casa há } 7 \text { portas e } 3 \text { chaves. Quantas portas há a mais do } \\
\text { que chaves? }\end{array}$ \\
\hline & & Subtração & $\begin{array}{l}\text { Numa festa o Tiago recebeu } 5 \text { carrinhos e o Rui recebeu } 2 \\
\text { aviões. Quantos aviões há a menos do que carros? }\end{array}$ \\
\hline & \multirow{2}{*}{ Comparado } & Adição & $\begin{array}{l}\text { A Maria tem } 3 \text { flores. A Rita tem mais } 2 \text { do que a Maria. } \\
\text { Quantas flores tem a Rita? }\end{array}$ \\
\hline & & Subtração & $\begin{array}{l}\text { A Laura tem } 5 \text { bonecas. A Rosa tem menos } 2 \text { do que a Laura. } \\
\text { Quantas bonecas tem a Rosa? }\end{array}$ \\
\hline & \multirow{2}{*}{ Referente } & Adição & $\begin{array}{l}\text { O Paulo tem mais } 2 \text { carros do que o Tiago. O Tiago tem } 4 . \\
\text { Quantos carros tem o Paulo? }\end{array}$ \\
\hline & & Subtração & $\begin{array}{l}\text { O João tem menos } 2 \text { balões do que o Miguel. O Miguel tem } 7 \\
\text { balões. Quantos balões tem o João? }\end{array}$ \\
\hline
\end{tabular}

Fonte: SOUTINHO (2016), adaptado de VERGNAUD (1982).

Cada criança foi entrevistada individualmente, no seu Jardim de Infância, numa sala cedida para o efeito. A ordem das questões da entrevista foi pré-estabelecida, igual para todas as crianças e alternando os tipos de problemas, tendo sido garantido, de acordo com o teste realizado previamente, que as crianças não eram afetadas por algum cansaço ou saturação que comprometesse os resultados. Para cada problema foi disponibilizado material figurativo alusivo à história apresentada, para que pudessem manipular caso necessitassem. No final de cada resolução foi-lhes solicitado que explicassem o seu procedimento e justificassem a sua resposta. As entrevistas foram conduzidas por uma investigadora, elemento externo à sala das crianças e uma das autoras deste artigo. Os dados foram recolhidos com recurso a gravação de vídeo e a notas de campo da investigadora. A análise dos dados foi realizada com recurso ao software Statistical Package for Social Sciences (SPSS), versão 19.0.

\section{Resultados}

Para se compreender como raciocinam as crianças durante a resolução dos problemas 
propostos, analisaram-se os seus desempenhos e as estratégias utilizadas nas respostas certas.

\section{Desempenho das crianças na resolução dos problemas propostos}

Foi atribuído 1 ponto a cada resposta correta aos problemas propostos. A cada resposta errada atribuíram-se 0 pontos. Registrou-se a pontuação de cada criança na resolução de cada problema. A Tabela 2 resume a média de respostas corretas nos problemas propostos, de acordo com o tipo de problema e a idade das crianças.

Tabela 2 - Média de proporções (desvio padrão) de respostas corretas

\begin{tabular}{lccc}
\hline \multirow{2}{*}{ TIPO DE PROBLEMAS } & \multicolumn{3}{c}{ MÉDIA (desvio padrão) } \\
\cline { 2 - 4 } & $\begin{array}{c}\mathbf{4} \text { anos } \\
(\mathrm{n}=30)\end{array}$ & $\begin{array}{c}\mathbf{5} \text { anos } \\
(\mathrm{n}=30)\end{array}$ & $\begin{array}{c}\mathbf{6} \text { anos } \\
(\mathrm{n}=30)\end{array}$ \\
\hline Composição de Duas Medidas & $.41(.29)$ & $.48(.25)$ & $.74(.22)$ \\
\hline Transformação Ligando Duas Medidas & $.42(.21)$ & $.51(.23)$ & $.72(.19)$ \\
\hline Relação Estática Ligando Duas Medidas & $.06(.15)$ & $.13(.19)$ & $.37(.31)$ \\
\hline \multicolumn{4}{c}{ Fonte: SOUTINHO (2016). }
\end{tabular}

Os problemas de Relação Estática Ligando Duas Medidas foram os mais difíceis de resolver por todas as crianças, tendo sido os problemas de Composição de Duas Medidas e os de Transformação Ligando Duas Medidas os mais acessíveis. Registra-se ainda que as crianças mais velhas têm um desempenho superior às mais novas nos três tipos de problemas, no entanto, tanto as crianças de 5 , como as de 4 anos, conseguem resolver corretamente muitos dos problemas propostos.

Nos problemas de Composição de Duas Medidas, observa-se que a maior parte das crianças de 6 anos acertou mais de metade dos problemas e salienta-se uma criança de 4 anos que acertou a totalidade das tarefas propostas para este tipo de problema. Ainda assim, seis crianças desta idade não acertaram nenhum problema deste tipo. De referir que a maioria das crianças de 5 anos resolveu com sucesso pelo menos metade dos problemas propostos.

Nos problemas de Transformação Ligando Duas Medidas, verifica-se que todas as crianças de 4 e 5 anos conseguiram resolver corretamente pelo menos um problema deste tipo e as crianças de 6 anos resolveram corretamente pelo menos 5 problemas. Observa-se que quatro crianças de 4 anos resolveram com sucesso $75 \%$ dos problemas apresentados, cinco crianças de 6 anos acertaram 100\% dos problemas propostos e metade das crianças de 5 anos responderam corretamente a mais $50 \%$ dos problemas propostos.

Os problemas de Relação Estática Ligando Duas Medidas foram os mais difíceis de resolver por crianças desta idade. Apesar do baixo sucesso observado em todas as idades, dáse conta de uma criança de 4 anos que conseguiu resolver corretamente 9 dos 12 problemas 
apresentados, o que corresponde a $75 \%$ dos problemas propostos, e 9 crianças de 6 anos que resolveram corretamente mais de metade dos problemas, havendo mesmo uma criança desta idade que apresentou $100 \%$ de resoluções corretas em todos os problemas propostos.

O teste de amostras independentes de Kruskal-Wallis (MARÔCO, 2010) sugere a existência de diferenças significativas no desempenho das crianças, em todos os tipos de problemas, de acordo com a idade. Esta diferença verifica-se entre os 4 e os 6 anos (problemas de Composição de Duas Medidas, $\chi_{k w}^{2}=-28,050, p<.001$; problemas de Transformação Ligando Duas Medidas, $\chi_{k w}^{2}=-31,233, p<.001$; problemas de Relação Estática Ligando Duas Medidas, $\chi_{k w}^{2}=-30,617, p<.001$ ) e entre os 5 e os 6 anos (problemas de Composição de Duas Medidas, $\chi_{k w}^{2}=-23,150, p<.05$; problemas de Transformação Ligando Duas Medidas, $\chi_{k w}^{2}=-20,717, p<.05$; problemas de Relação Estática Ligando Duas Medidas, $\left.\chi_{k w}^{2}=-21,733, p<.05\right)$. Entre os 4 e os 5 anos não se registram diferenças significativas, em nenhum dos tipos de problemas.

Estudos sugerem que enunciados que refiram claramente a operação requerida para a solução são considerados mais fáceis do que aqueles em que é necessário o reconhecimento da relação inversa entre as operações. Assim, conduziu-se uma análise mais detalhada de forma a perceber as diferenças de desempenho das crianças nos três tipos de problemas de estrutura aditiva, de acordo com o elemento desconhecido. O resumo da média de proporções de respostas corretas, nos diferentes tipos de problemas de estrutura aditiva, de acordo com o elemento desconhecido e a idade das crianças é apresentado na Tabela 3.

Apesar da facilidade com que as crianças resolveram os problemas de Composição de Duas Medidas, assumem-se como mais difíceis de resolver os problemas cujo elemento desconhecido é a parte, observando-se um maior número de crianças a errar estes problemas (10 crianças de 6 anos, 18 de 5 anos e 22 de 4 anos). Já os problemas de Composição de Duas Medidas com o todo desconhecido foram resolvidos com grande sucesso pela maioria das crianças, registrando-se que apenas 10 crianças, das 90 participantes não conseguiram resolver nenhum problema deste tipo, sendo que dessas, 7 tinham apenas 4 anos.

Tabela 3 - Média de proporções (desvio padrão) de respostas corretas de acordo com o elemento desconhecido

\begin{tabular}{lllcc}
\hline \multirow{2}{*}{$\begin{array}{c}\text { TIPO DE } \\
\text { PROBLEMAS }\end{array}$} & \multicolumn{1}{c}{\begin{tabular}{c} 
Elemento \\
\cline { 2 - 5 }
\end{tabular}} & $\begin{array}{c}\text { MescónIA (desvio padrão) } \\
(\mathrm{n}=30)\end{array}$ & $\begin{array}{c}\mathbf{5} \text { anos } \\
(\mathrm{n}=30)\end{array}$ & $\begin{array}{c}\mathbf{6} \text { anos } \\
(\mathrm{n}=30)\end{array}$ \\
\hline $\begin{array}{l}\text { Composição de Duas } \\
\text { Medidas }\end{array}$ & Parte & $.15(.27)$ & $.22(.28)$ & $.52(.43)$ \\
\cline { 2 - 5 } & Todo & $.67(.42)$ & $.75(.34)$ & $.97(.13)$ \\
\hline Transformação & Resultado & $.67(.28)$ & $.79(.30)$ & $.90(.18)$ \\
\hline
\end{tabular}




\begin{tabular}{llccc}
\hline $\begin{array}{l}\text { Ligando Duas } \\
\text { Medidas }\end{array}$ & Transformação & $.38(.31)$ & $.45(.30)$ & $.70(.21)$ \\
\cline { 2 - 5 } & Início & $.23(.27)$ & $.29(.26)$ & $.57(.32)$ \\
\hline $\begin{array}{l}\text { Relação Estática } \\
\text { Ligando Duas } \\
\text { Medidas }\end{array}$ & Diferença & $.03(.11)$ & $.17(.27)$ & $.39(40)$ \\
\cline { 2 - 5 } & Comparado & $.08(.20)$ & $.17(.30)$ & $.44(.40)$ \\
\cline { 2 - 5 } & Referente & $.06(.17)$ & $.04(.12)$ & $.27(.31)$ \\
\hline
\end{tabular}

Fonte: SOUTINHO (2016).

Nos problemas de Transformação Ligando Duas Medidas, os que obtiveram maior nível de sucesso foram os problemas cujo elemento ausente era o resultado, registrando crianças a acertar a totalidade destes problemas. Os problemas com o início desconhecido foram aqueles nos quais se observaram maiores taxas de insucesso, com valores médios inferiores a 30\% no grupo das crianças de 4 anos. Apenas $20 \%$ das crianças de 6 anos resolveram corretamente a totalidade dos problemas de Transformação Ligando Duas Medidas com o início desconhecido. Nos problemas de Transformação Ligando Duas Medidas com a transformação desconhecida as crianças acertaram pelo menos metade dos problemas propostos. E apesar de se registrarem casos de crianças de 4 e 5 anos a errarem todos estes problemas, também se observam crianças de 4, 5 e 6 anos a resolver corretamente a totalidade destes problemas.

Os problemas de Relação Estática Ligando Duas Medidas foram o que apresentaram menores níveis de sucesso, quando comparados com os problemas de Composição de Duas Medidas e os de Transformação Ligando Duas Medidas. No entanto, em todos os grupos etários houve crianças que resolveram corretamente este tipo de problemas, independentemente do elemento desconhecido. Neste tipo de problemas, os que apresentaram maiores taxas de sucesso foram os problemas com o elemento comparado desconhecido, nos quais se observam 36 crianças, das 90 participantes, a resolverem corretamente todos os problemas propostos. Os que foram considerados como mais difíceis foram os problemas com o referente desconhecido, mesmo para as crianças com 6 anos.

De acordo com o teste de Kruskal-Wallis (MARÔCO, 2010), observa-se que o desempenho das crianças é influenciado pela idade, independentemente do elemento desconhecido. Crianças mais velhas, de 6 anos, têm melhor desempenho do que as crianças mais novas, 4 e 5 anos. Em todos tipos de problemas, as diferenças estatisticamente significativas ocorrem entre as crianças de 4 e 6 anos (Composição de Duas Medidas: todo desconhecido, $\chi_{k w}^{2}(2)=22.067, p<.005$; parte desconhecida, $\chi_{k w}^{2}(2)=17.817, p<.005$; Transformação Ligando Duas Medidas: resultado desconhecido, $\chi_{k w}^{2}(2)=21.100, p<.005$; transformação desconhecida, $\chi_{k w}^{2}(2)=26.983, p<.001$; início desconhecido, $\chi_{k w}^{2}(2)=26.250$, 
$p<.001$; Relação Estática Ligando Duas Medidas: diferença desconhecida, $\chi_{k w}^{2}(2)=26.617$, $p<.001$; comparado desconhecido, $\chi_{k w}^{2}(2)=22.583, p<.001$; referente desconhecido, $\chi_{k w}^{2}(2)=$ $17.567, p<.005)$.

Ocorrem também entre as crianças de 5 e 6 anos em todos os problemas (Composição de Duas Medidas: todo desconhecido, $\chi_{k w}^{2}(2)=16.933, p<.005$; parte desconhecida, $\chi_{k w}^{2}(2)=$ 14.683, $p<.005$; Transformação Ligando Duas Medidas: transformação desconhecida, $\chi_{k w}^{2}(2)$ $=21.367, p<.005$; início desconhecido, $\chi_{k w}^{2}(2)=20.650, p<.005$; Relação Estática Ligando Duas Medidas: diferença desconhecida, $\chi_{k w}^{2}(2)=15.533, p<.005$; comparado desconhecido, $\chi_{k w}^{2}(2)=17.117, p<.005$; referente desconhecido, $\left.\chi_{k w}^{2}(2)=18.133, p<.005\right)$. Entre os 4 e os 5 anos não se registram diferenças significativas.

Em suma, pode dizer-se que crianças tão pequenas como as de 4 aos 6 anos conseguem resolver com sucesso alguns problemas de Composição de Duas Medidas, de Transformação Ligando Duas Medidas e de Relação Estática Ligando Duas Medidas. Os problemas de Composição de Duas Medidas foram os mais fáceis para as crianças, seguindose os de Transformação Ligando Duas Medidas, sendo os problemas de Relação Estática Ligando Duas Medidas os mais difíceis. As crianças mais velhas têm um desempenho superior às mais novas em todos os tipos de problemas, não havendo diferenças significativas entre as crianças de 4 e 5 anos.

Procurando conhecer melhor como raciocinam as crianças pequenas quando resolvem corretamente os problemas propostos, analisaram-se as estratégias por elas adotadas na resolução correta dos problemas propostos.

\section{Estratégias de resolução dos problemas propostos}

As estratégias observadas durante a resolução dos problemas que conduziram a respostas corretas possibilitaram uma categorização distinguindo, à semelhança de Carpenter et al. (1999), três grandes grupos: estratégias de manipulação direta, estratégias de contagem e estratégias com fatos numéricos. As estratégias de manipulação direta refletem a manipulação dos objetos pelas crianças, formando conjuntos com as quantidades enunciadas nos problemas; as estratégias de contagem são aplicadas quando a criança resolve o problema pela contagem, sem a manipulação dos objetos; as estratégias com fatos numéricos são consideradas quando a criança apela a fatos conhecidos já memorizados, como, por exemplo, $3+3,4+4$ ou ainda outras $4+2$, ou seja, quando domina a composição de determinado número 
pelas diferentes partes possíveis que o compõem.

Foi criada a categoria "Inconclusivo" para os casos em que as estratégias usadas pelas crianças não conseguem determinar uma forma de atuar, apesar de conduzirem a respostas corretas. A Tabela 4 regista o tipo de estratégias usadas pelas crianças na resolução correta dos problemas apresentados.

Tabela 4 - Tipo de estratégias observadas na resolução de problemas

\begin{tabular}{|c|c|c|c|c|c|c|c|c|c|}
\hline \multirow[b]{3}{*}{$\begin{array}{c}\text { TIPO DE } \\
\text { ESTRATÉGIAS }\end{array}$} & \multicolumn{9}{|c|}{ TIPO DE PROBLEMAS } \\
\hline & \multicolumn{3}{|c|}{$\begin{array}{c}\text { Composição de Duas } \\
\text { Medidas }\end{array}$} & \multicolumn{3}{|c|}{$\begin{array}{c}\text { Transformação Ligando } \\
\text { Duas Medidas }\end{array}$} & \multicolumn{3}{|c|}{$\begin{array}{c}\text { Relação Estática Ligando } \\
\text { Duas Medidas }\end{array}$} \\
\hline & $\begin{array}{c}4 \text { anos } \\
(\%)\end{array}$ & $\begin{array}{c}5 \text { anos } \\
(\%)\end{array}$ & $\begin{array}{c}6 \text { anos } \\
(\%)\end{array}$ & $\begin{array}{c}4 \text { anos } \\
(\%)\end{array}$ & $\begin{array}{c}5 \text { anos } \\
(\%)\end{array}$ & $\begin{array}{c}6 \text { anos } \\
(\%)\end{array}$ & $\begin{array}{c}4 \text { anos } \\
(\%)\end{array}$ & $\begin{array}{c}5 \text { anos } \\
(\%)\end{array}$ & $\begin{array}{c}6 \text { anos } \\
(\%)\end{array}$ \\
\hline Manipulação Direta & 100 & 84.7 & 67.5 & 86.8 & 81.6 & 72.6 & 57.1 & 71.1 & 68.2 \\
\hline Contagem & - & 6.8 & 11.1 & 0.7 & 3.8 & 10.1 & 9.5 & 11.1 & 20.4 \\
\hline Fatos Numéricos & - & 5.1 & 18 & 1.3 & 6.5 & 10.8 & - & 8.9 & 3.8 \\
\hline Inconclusivo & - & 3.4 & 3.4 & 11.2 & 8.1 & 6.5 & 33.4 & 8.9 & 7.6 \\
\hline
\end{tabular}

A Tabela 4 sugere que à medida que a idade das crianças vai aumentando, diminui o recurso às estratégias de manipulação direta, mas aumenta o recurso a estratégias mais abstratas, como estratégias de contagem ou com fatos numéricos. As crianças de 5 e 6 anos já resolvem muitos problemas recorrendo a estratégias de contagem e estratégias com factos numéricos. A maior percentagem de estratégias inconclusivas e das quais resultaram respostas corretas, situam-se no grupo das crianças de 4 anos, o que poderá indicar que estas crianças, conseguindo chegar a um resultado correto, ainda estão a afinar o seu procedimento.

Nas estratégias de manipulação direta, foram observadas distintas formas de manipular os objetos representativos das quantidades do enunciado, algumas apresentadas por Carpenter et al. (1999), outras criadas pelas autoras, por ainda não terem sido descritas na literatura.

Na categoria "Juntar Tudo" a criança dispõe os elementos dos dois conjuntos dados e conta todos para saber o resultado. No problema "A cadelinha da Inês teve cachorrinhos: 5 branquinhos e 3 castanhos. Quantos cachorrinhos teve a cadelinha da Inês?", por exemplo, a criança coloca o conjunto dos cães brancos e o conjunto dos cães castanhos, conta todos e responde o resultado da contagem "oito" (ver Figura 1).

Na categoria "Juntar Para" a criança faz um conjunto com a quantidade inicial e vai adicionando elementos até que o novo conjunto atinja a quantidade total dada no problema. A resposta é dada contando os elementos adicionados ao conjunto inicial. Por exemplo, no problema "O Pedro apanhou 3 gafanhotos na relva e mais alguns na areia. Agora ele tem 5 gafanhotos. Quantos gafanhotos apanhou na areia?", a criança coloca o conjunto de três gafanhotos, quantidade mencionada inicialmente e junta mais dois gafanhotos até perfazer cinco elementos no total, quantidade total mencionada no problema, e conta de seguida os que 
adicionou (ver Figura 2).

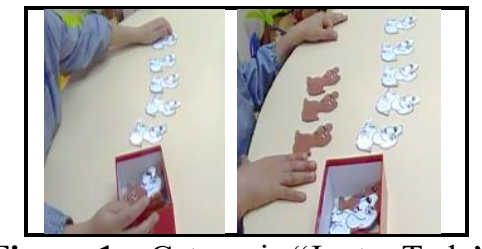

Figura 1 - Categoria "Juntar Tudo"

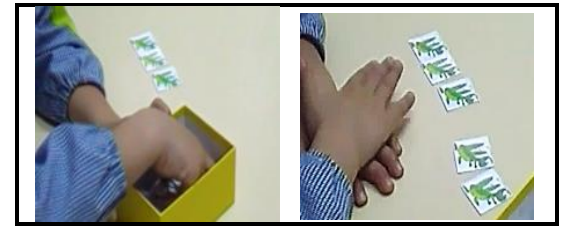

Figura 2 - Categoria "Juntar Para"

Fonte: CARPENTER et al. (1999).

$\mathrm{Na}$ categoria "Separar de" a criança coloca um conjunto com a quantidade maior mencionada no problema, que corresponde ao resultado, e retira deste a quantidade menor dita, contando os elementos que sobram para saber a resposta. No problema "A Maria tinha 6 flores, deu 2 à sua mãe. Quantas flores tem ela agora?”, por exemplo, a criança coloca seis flores, a quantidade maior que é mencionada no problema, e retira deste conjunto as duas flores, que correspondem à quantidade menor mencionada, contando, de seguida, as flores que restam (ver Figura 3).

Na estratégia "Separar Para" a criança faz o conjunto maior dito no enunciado e retira os elementos necessários até deixar ficar a quantidade menor mencionada no problema. A resposta é encontrada pela quantidade que foi retirada, como, por exemplo, no problema "A mãe do Pedro fez 7 bolos para a festa. O Pedro comeu alguns em segredo e agora a mãe só tem 4 bolos para pôr na mesa. Quantos bolos comeu o Pedro?”, a criança coloca um conjunto de 7 bolos (ver Figura 4), a quantidade maior mencionada no problema, e retira os elementos necessários para que fiquem apenas quatro bolos, a quantidade menor mencionada, contando de seguida os bolos que retirou.

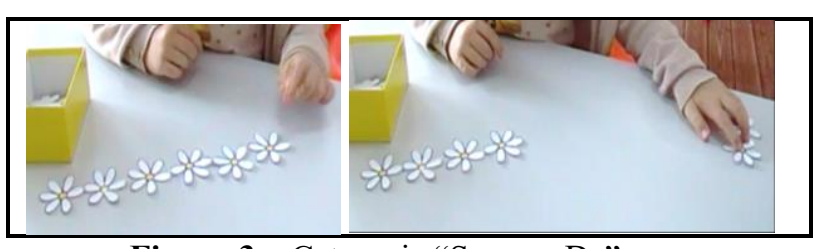

Figura 3 - Categoria "Separar De"

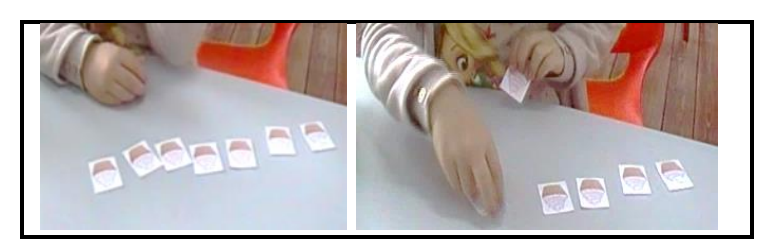

Figura 4 - Categoria "Separar Para"

Fonte: CARPENTER et al. (1999).

Na estratégia “Correspondência termo a termo", usada para resolver problemas de Relação Estática Ligando Duas Medidas, a criança coloca os elementos dos dois conjuntos em correspondência um-a-um, até que os elementos de um dos conjuntos se esgote e ela possa contar os elementos sobrantes, de forma a encontrar a diferença. Por exemplo, no problema “Na sala da Bela há 5 meninos e 9 meninas. Quantas meninas há a mais do que meninos?”, a criança coloca nove meninas em linha, e em seguida coloca cinco meninos por cima das meninas, em correspondência um-a-um e conta as meninas que não têm correspondência (ver Figura 5). 
A categoria "Correspondência Separando" foi criada para contemplar os casos em que a criança, na resolução dos problemas de Relação Estática Ligando Duas Medidas, faz corresponder um-a-um os elementos de um conjunto ao do outro, mas deixa em aberto/vazio, a diferença. A resposta é encontrada contando os elementos do conjunto menor, como, por exemplo, no problema “A Bruna tem na mochila 3 bananas a menos do que a Rosa, que tem 7. Quantas bananas tem a Bruna na mochila?”, a criança coloca 7 bananas, que é a quantidade mencionada como referência, a quantidade do elemento comparado, e estabelece a correspondência um-a-um com as 4 bananas, quantidade que corresponde ao elemento comparado, e verifica o número de bananas que não têm correspondência (ver Figura 6).

A diferença entre esta estratégia e a estratégia de Correspondência termo a termo é que, nesta última, a criança é conhecedora das quantidades dos dois conjuntos e a sua atividade consiste em colocar as duas quantidades em correspondência, enquanto que na Correspondência Separando a criança vai colocando os elementos do segundo conjunto, que não sabe quantos são, em correspondência com o primeiro conjunto, tendo em atenção e contando os elementos que ficam sem correspondência. Quando os elementos que ficam sem correspondência tiverem a quantidade mencionada no problema, que corresponde à diferença, a criança para de colocar elementos no segundo conjunto e verifica quantos colocou.

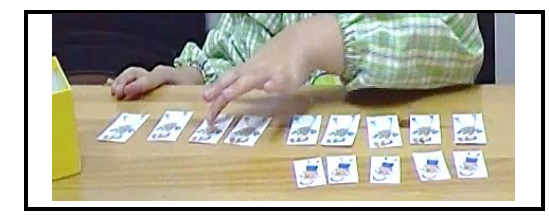

Figura 5 - Categoria "Correspondência termo a termo". Figura 6 - Categoria "Correspondência Separando". Fonte: SOUTINHO (2016).

A categoria "Correspondência Juntando" foi criada para contemplar os casos em que, para resolver problemas de Relação Estática Ligando Duas Medidas, a criança estabelece a correspondência um-a-um entre os dois conjuntos e adiciona a diferença ao segundo conjunto, sendo a resposta encontrada pela quantidade do conjunto maior. No problema "A Maria tem 3 flores. A Rita tem mais 2 do que a Maria. Quantas flores tem a Rita?”, por exemplo, a criança coloca três flores que correspondem à quantidade mencionada de um dos conjuntos e coloca um outro conjunto de flores por cima do primeiro, em correspondência um-a-um e adiciona a este último mais duas flores, que corresponde à diferença entre os conjuntos, contando de seguida as flores que estão no conjunto maior (ver Figura 7). 


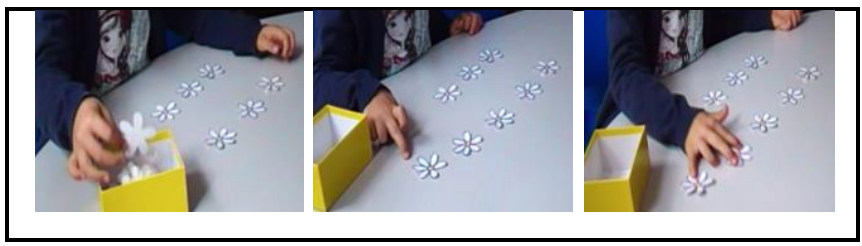

Figura 7 - Categoria "Correspondência Juntando"

Fonte: SOUTINHO (2016).

A categoria "Correspondência Para" foi criada tendo sido observado casos em que a criança coloca os dois conjuntos mencionados no problema, mas sem estabelecer a correspondência um-a-um. No segundo conjunto, a criança conta a quantidade mencionada no primeiro conjunto, ou seja, o mesmo número de elementos do primeiro conjunto e retira o excedente, contando quantos elementos retirou. No problema "Numa casa há 7 portas e 3 chaves. Quantas portas há a mais do que chaves?”, por exemplo, a criança coloca sete portas e três chaves, correspondentes aos dois conjuntos mencionados no problema, de seguida conta três portas, correspondente ao número existente de chaves e conta as portas que restam, não estabelecendo para tal correspondência um-a-um (ver Figura 8).

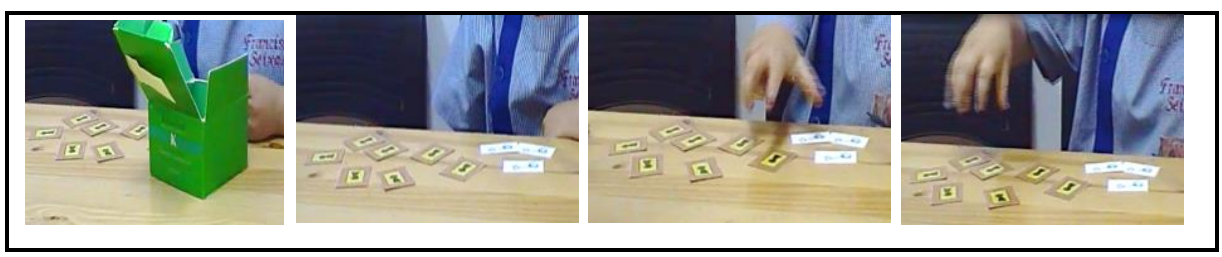

Figura 8 - Categoria "Correspondência Para" Fonte: SOUTINHO (2016).

A categoria “A-Mais Correspondência” foi criada para considerar os casos em que, na resolução dos problemas de Relação Estática Ligando Duas Medidas, a criança coloca o primeiro conjunto, em seguida coloca, num plano superior e fora de correspondência, a diferença, ou seja, os que estão a mais, a seguir coloca os elementos do outro conjunto em correspondência um-a-um com o primeiro e conta o conjunto maior, como, por exemplo, na resolução do problema "A mamã deu à Joana 4 bolos para o lanche e deu ao Diogo mais 2 do que à Joana. Quantos bolos deu ao Diogo?”, a criança coloca quatro bolos em linha, que corresponde ao número de elementos do primeiro conjunto, em seguida coloca dois bolos na fila acima e fora do espaço de correspondência relativos à diferença, e, por último, coloca em correspondência um-a-um os quatro bolos que restam ao segundo conjunto, completando a fila onde já estavam os dois bolos que representavam a diferença, e conta este último conjunto (ver Figura 9). 


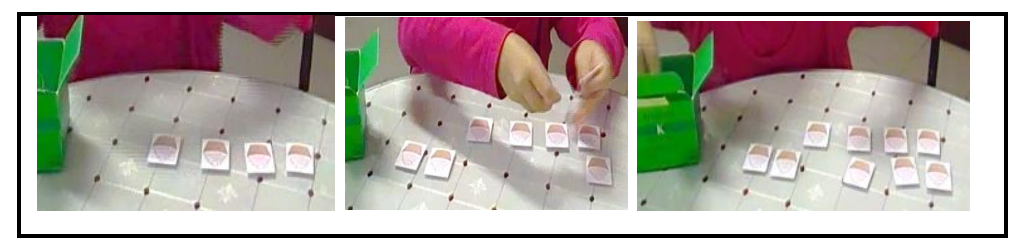

Figura 9 - Categoria "A-Mais Correspondência" Fonte: SOUTINHO (2016).

As estratégias de manipulação direta usadas pelas crianças parecem variar, não de acordo com a idade, mas sobretudo consoante o tipo de problemas. Nos três grupos etários, as crianças recorrem à mesma variedade de estratégias, escolhendo estratégias diferentes de acordo com o tipo de problemas que pretendem resolver. Verifica-se também que as estratégias de correspondência são apenas usadas nos problemas de Relação Estática Ligando Duas Medidas, em que manipulam dois conjuntos distintos, estabelecendo, de alguma forma, uma correspondência entre ambos, enquanto que nos problemas de Composição de Duas Medidas e Transformação Ligando Duas Medidas a manipulação é feita num só conjunto, no qual juntam ou separam elementos.

Nas estratégias de contagem foram consideradas as categorias: Contando do Primeiro; Contando do Maior; Contando do Menor; Contando Até; Contagem Decrescente; Contagem Decrescente Até.

Na categoria "Contando do Primeiro" a criança conta a partir da primeira quantidade mencionada no problema e a sequência de contagem termina quando o número de elementos adicionado está completo. No problema "Numa árvore estavam 3 macaquinhos em pé e 4 de cabeça para baixo. Quantos macaquinhos estão ao todo na árvore?", a criança explica que "já tinha 3 [mostra 3 dedos, faz uma pausa e prossegue depois a contagem levantando os restantes dedos] 4, 5, 6, 7" (Entrevista, 2016).

Na categoria "Contando do Maior", a contagem é iniciada a partir da quantidade maior mencionada no problema. Na resolução do mesmo problema, a criança diz "estavam 4 , e depois contei assim: 5 [levanta um dedo], 6 [levanta outro dedo], 7 [levanta outro dedo e pára]" (Entrevista, 2016). Na categoria "Contando do Menor”, a criança começa a contagem na quantidade menor que é mencionada no problema e termina quando adiciona o número maior dito no enunciado. Na resolução do problema anteriormente descrito, a criança diz: "estavam 3 [faz uma pausa e continua a contagem a partir desse número], 4 [levanta um dedo], 5 [levanta outro dedo], 6 [levanta outro dedo], 7 [levanta o 4. ${ }^{\circ}$ dedo]" (Entrevista, 2016).

Na categoria "Contando Até", a criança começa a contagem pelo elemento menor apresentado no problema e vai contando até dizer o número maior apresentado, sendo a 
resposta determinada pelo número de passos na contagem. No problema "A Joana tinha algumas bonecas, a tia deu-lhe mais 3 e agora ela tem 8. Quantas bonecas tinha a Joana no início?”, a criança afirma que "ela tinha 3, estas já desapareceram [mostra os 3 dedos que depois volta a baixar], 4 [levanta um dedo], 5 [levanta outro dedo], 6 [levanta outro dedo], 7 [levanta outro dedo], e 8 [levanta o último dedo], 5 [refere mostrando os 5 dedos levantados]" (Entrevista, 2016).

$\mathrm{Na}$ categoria "Contagem Decrescente" a criança realiza a contagem regressiva, menciona tantos passos na contagem quantos a quantidade que é retirada e responde o último número dito. No problema "A Rosa tem 8 morangos colocados em 2 taças. Uma taça tem 5 morangos, quantos morangos tem a outra taça?", a criança diz "8 [pausa], 7 [mostra a mão com os dedos abertos e baixa um dedo], 6 [baixa outro dedo], 5 [baixa outro dedo], 4 [baixa outro dedo], 3 [baixa outro dedo e neste momento tem a mão toda fechada] 3, são 3" (Entrevista, 2016).

A categoria "Contagem Decrescente Até" compreende os casos em que a criança realiza a contagem regressiva, mas a contagem para quando o menor número mencionado no problema é dito, e a resposta é obtida pelo número de passos na contagem. No problema "A mãe do Pedro fez 7 bolos para a festa. O Pedro comeu alguns em segredo e agora a mãe só tem 4 bolos para pôr na mesa. Quantos bolos comeu o Pedro?”, a criança levanta 7 dedos e vai baixando um a um até ter baixado os 4 dedos, enquanto faz a contagem decrescente " 7 [pausa e baixa um dedo], 6 [baixa um dedo], 5 [baixa outro dedo], 4 [baixa outro dedo e deixa 3 dedos levantados], são 3" (Entrevista, 2016).

O recurso a estratégias de contagem é mais frequente em crianças de 6 anos e ocorre maioritariamente nos problemas de Transformação Ligando Duas Medidas. No entanto, é de notar o recurso a este tipo de estratégias, que envolvem já algum grau de abstração, por crianças tão novas quanto as de 4 e 5 anos. É de salientar ainda o uso da Contagem Decrescente por crianças de 6 anos, estratégia que, de acordo com Fuson (1992) é mais difícil, e por isso, pouco habitual em crianças tão pequenas.

Nas estratégias com fatos numéricos, as crianças apelam a fatos e combinações aprendidos, não manipulam objetos nem usam a contagem para resolver os problemas propostos. Foi identificada a categoria "Fatos Numéricos", na qual a criança se serve de combinações de números aprendidas, como por exemplo $3+3,4+4$ ou ainda outras $4+2$, o que revela que mentalmente domina a decomposição de alguns números. Quando, perante o problema "A Ana tinha algumas amêndoas, deu 3 à sua mãe e ficou com 2. Quantas amêndoas tinha a Ana no início?”, responde “5”, sem manipular nenhum objeto, nem realizar nenhuma 
contagem, e acrescenta "porque 2 mais 3 é 5" (Entrevista, 2016).

Identificou-se também a categoria "Fatos Derivados" que compreende os casos em que a criança se serve de fatos numéricos conhecidos para encontrar outras combinações de números. No problema "A Rosa tem 8 morangos colocados em 2 taças. Uma taça tem 5 morangos, quantos morangos tem a outra taça?", a criança responde "3" e acrescenta para explicar "porque metade de 8 é 4 e se tem 5 já roubou 1 ao de 3" [o seu raciocínio pode ser traduzido da seguinte forma: $8=4+4$, logo se um grupo tem mais 1 , o outro tem menos 1 , se o grupo que tinha 4 tem menos 1, fica com 3] (Entrevista, 2016). Apesar da estratégia com Fatos Numéricos ser considerada uma estratégia que requer um elevado grau de abstração, mais do que as estratégias de contagem (Carpenter et al., 1999), é notório o seu uso por crianças tão novas quanto as deste estudo.

\section{Comentários Finais}

À semelhança de estudos prévios realizados em contextos internacionais com crianças mais velhas (ver CARPENTER \& MOSER, 1984), também o nosso estudo, conduzido com crianças pequenas (4-6 anos), revelou que estas resolvem com sucesso alguns problemas de raciocínio aditivo.

Estudos prévios com crianças mais velhas mostram que os problemas de Composição de Duas Medidas e os problemas de Transformação Ligando Duas Medidas são mais facilmente resolvidos do que os problemas de Relação Estática Ligando Duas Medidas (ver DE CORTE \& VERSCHAFFEL, 1987; VERGNAUD, 1982). A dificuldade apontada na resolução destes problemas parece justificar-se com a interpretação que as crianças fazem das relações que se estabelecem nas situações, logo, do cálculo relacional que têm que realizar para escolher a operação necessária à resolução (VERGNAUD, 2011; NUNES; BRYANT; WATSON, 2009).

Uma outra causa apontada como justificativa da dificuldade na resolução dos problemas de Relação Estática Ligando Duas Medidas são as incongruências linguísticas, que conduzem ao erro quando não apreciadas devidamente. A expressão comparativa "a mais" ou "a menos" é muitas vezes interpretada pela criança como uma transformação da quantidade inicial, de adição (mais), ou de subtração (menos), o que não traduz a essência da situação descrita, uma situação em que os valores se referem à relação entre as quantidades.

Este estudo mostra que as crianças mais pequenas (4-6 anos) manifestam já maior facilidade na resolução dos problemas Composição de Duas Medidas e dos problemas de 
Transformação Ligando Duas Medidas do que na resolução de problemas Relação Estática Ligando Duas Medidas. Fato que talvez se deva à frequência com que estas situações se apresentam no dia a dia das crianças, havendo uma maior facilidade de interpretação linguística das relações descritas nos enunciados. É de salientar, contudo, que crianças tão pequenas quanto as de 4 anos tenham conseguido resolver corretamente problemas nos quais a operação requerida na resolução era inversa à mencionada no problema, situações verificadas sobretudo nos problemas de Transformação Ligando Duas Medidas com o início desconhecido e nos problemas de Composição de duas Medidas com a parte desconhecida.

Sendo espectável que as crianças mais velhas obtivessem melhores resultados, é de evidenciar o êxito alcançado pelas crianças tão pequenas quanto as de 4 e 5 anos na resolução dos problemas, sendo que alguns deles exigem um pensamento mais elaborado, uma vez que a operação requerida é inversa à mencionada, como já apontado nas situações não prototípicas. Apesar dos problemas de Relação Estática Ligando Duas Medidas se mostrarem mais difíceis de resolver, foi possível registrar sucesso nas crianças com idades inferiores a 6 anos, o que é digno de destaque, atendendo às dificuldades inerentes a este tipo de problema.

Nestes problemas, as crianças recorrem a uma diversidade de estratégias, algumas tão elaboradas e ainda não descritas na literatura. Apesar da maioria das estratégias observadas neste estudo terem sido estratégias de manipulação de objetos que estavam à disposição, é interessante observar que crianças de 4 anos tenham resolvido problemas recorrendo a estratégias de contagem e mesmo a estratégias com fatos numéricos. Para Carpenter et al. (1999), as crianças vão evoluindo no uso de estratégias, estratégias de modelação direta vão dando lugar a estratégias mais abstratas como as de contagem e estas, por sua vez, dão lugar a outras ainda mais abstratas, as estratégias com fatos numéricos. Não sendo possível a generalização destes resultados, importa ressaltar que é possível às crianças de 4 anos usarem estratégias mais abstratas do que a simples modelação direta para resolverem os problemas.

Tal como há uma grande variedade de procedimentos que as crianças adotam para resolver problemas de Composição de Duas Medidas e Transformação Ligando Duas Medidas, também inventam muitas formas de atuar que melhor, e de uma forma mais eficiente, as conduza ao resultado correto nos problemas de Relação Estática Ligando Duas Medidas. A maior parte destes problemas foram resolvidos com diferentes estratégias de correspondência, identificadas nos procedimentos das crianças e ainda não descritos em estudos prévios.

Quando, para resolver um problema de Relação Estática Ligando Duas Medidas com o referente desconhecido, a criança cria uma estratégia em que coloca o conjunto tido como 
referência e vai estabelecendo uma correspondência termo-a-termo entre esses elementos e os do conjunto que pretende conhecer, mas deixa, desde logo, em aberto o espaço correspondente à diferença, e que é mencionada na situação (estratégia designada por “Correspondência Separando"), revela que percebe que uma das quantidades expressas no problema pertence a um conjunto e que a outra corresponde à relação que é estabelecida entre os dois conjuntos. Da mesma forma que, quando recorre a uma estratégia que aqui identificamos como "A-mais-correspondência" para resolver um problema com o elemento comparado desconhecido, identifica e coloca os elementos referentes à diferença entre os dois conjuntos do problema, percebe que as quantidades mencionadas no enunciado desempenham diferentes papéis, correspondendo uma a um conjunto e a outra à relação entre os dois conjuntos.

É já sabido que as crianças mais velhas conseguem resolver, com sucesso, problemas de estrutura aditiva. Este estudo permite afirmar que as crianças de 4, 5 e 6 anos, a frequentar a educação pré-escolar, demonstram um conhecimento informal que lhes permite resolver, com estratégias diversas e algumas até com elevado grau de abstração, determinados problemas de raciocínio aditivo. Talvez valha a pena repensar as práticas de educação préescolar, ajustando as tarefas a propor às crianças, desde os 4 anos de idade, que podem constituir veículo para o desenvolvimento do raciocínio lógico-dedutivo das crianças. Não se defende a imposição de tarefas, mas antes a apresentação das mesmas como adivinhas, jogos, ou pequenos problemas que facultem à criança a oportunidade de estimular o seu pensamento, brincando.

\section{Referências}

BAROODY, A.; GINSBURG, H. The relationship between initial meaningful and mechanical performance in mathematics. In: HIEBERT , J. (Ed.). Conceptual and Procedural Knowledge: The case of Mathematics. Hillsdale, NJ: Erlbaum, 1986. p. 75-112.

BECKER, J. P.; SELTER, C. Elementary school practices. In: BISHOP, A. et al. International Handbook of Mathematics Education. Dordrecht: Kluwer, 1994. p. 511-564.

CARPENTER, T.; MOSER, J. The acquisition of addition and subtraction concepts in grades one through three. Journal for Research in Mathematics Education, v. 3, n. 15, p. 179-202, 1984.

CARPENTER, T. et al. Children's Mathematics: cognitively guided instruction. USA: Leigh Peake, 1999.

DE CORTE, E.; VERSCHAFFEL, L. The effect of semantic structure on first graders' strategies for solving addition and subtraction word problems. Journal for Research in Mathematics Education, n. 18 , p. 363-381, 1987. 
FUSON, K. Relationships between counting and cardinality from age 2 to age 8 . In: JACQUELINE, B.; MELJAC, C.; FISCHER, J. P. Pathways to Number: Children's developing numerical abilities. Hillsdale, NJ, England: Lawrence Erlbaum Associates, 1992. p. 127-149.

GINSBURG, H.; SEO, K. H. Mathematics in children's thinking. Mathematical Thinking and Learning, New York, v. 1, n. 2, p. 113-129, 1999.

KAMII, C.; DOMINICK, A. To teach or not to teach algorithms. Journal of Mathematical Behavior, v. 1, n. 16, p. 51-61, 1997.

MARÔCO, J. Análise estatística com o PASW Statistics. Pêro Pinheiro: Report Number, 2010.

NUNES, T.; BRYANT, P. Crianças Fazendo Matemática. Porto Alegre: Artes Médicas, 1996.

NUNES, T.; BRYANT, P.; WATSON, A. Key Understandings in Mathematics Learning. Nuffield Foundation, 2009.

PIAGET, J.; SZEMINSKA, A. A Gênese do Número na Criança. Rio de Janeiro: Zahar Editores, 1971.

RESNICK, L. Developing mathematical knowledge. American Psychologist, Washington, v. 2, n. 44, p. 162-169, 1989.

SOUTINHO, F. A compreensão dos problemas de estrutura aditiva e estrutura multiplicativa por crianças do pré-escolar. 2016. 519f. Tese (Doutoramento em Ciências de Educação: Educação Matemática) - Instituto de Educação, Universidade do Minho, Braga, 2016.

VERGNAUD, G. A classification of cognitive tasks and operations of thought involved in addition and subtraction problems. In: CARPENTER, T. P.; MOSER, J. M.; ROMBERG, T. A. (Ed.). Addition and subtraction: A cognitive perspective. Hillsdale, NJ: Lawrence Erlbaum Ass., 1982. p. 60-67.

VERGNAUD, G. O longo e o curto prazo na aprendizagem da matemática. Educar em Revista, Curitiba, p. 15-27, 2011. 